\title{
Estudo da incidência de parasitas intestinais em verduras comercializadas em feira livre e supermercado de Londrina
}

\author{
Study of the incidence of intestinal parasites in vegetables \\ commercializes in free trade fair and supermarket Londrina
}

\author{
Priscila Ruzzon Nomura'; Ana Rúbia Magalhães Ferreira²; Rafael Algusto \\ Rafaelli³; Jessica Grande Augusto ${ }^{4}$; Vera Lucia Hideko Tatakihara5; Luiz Antonio \\ Custódio $^{6}$; Valter Abou Murad ${ }^{7}$
}

\begin{abstract}
Resumo
$\overline{\mathrm{O} \text { objetivo deste trabalho foi determinar a presença de parasitas intestinais em amostras de alfaces } \mathrm{e}}$ almeirões comercializadas em feira livre e supermercado da cidade de Londrina, Paraná. Foram coletadas e analisadas oito amostras de alface (Lactuca sativa) e oito amostras de almeirão (Cichirium intybus). A análise foi realizada no Laboratório de Extensão e Pesquisas em Enteroparasitoses - L.E.P.En. Foram encontrados cistos de Endolimax nana, Entamoeba coli, Entamoeba histolytica, Balantidium coli, larvas de Strongyloides stercoralis, larvas e ovos de Ancilostomídeos e ovos de Ascaris spp. Esses resultados demonstram a necessidade de medidas higiênico-sanitárias, visando à educação da comunidade, possibilitando a melhoria na condição de vida da população.
\end{abstract}

Palavras-chave: Parasitas intestinais. Verduras. Pesquisa. Saúde pública.

\begin{abstract}
The objective of this study was to determine the presence of intestinal parasites in samples of lettuce and chicory sold in street market and supermarket Londrina city, Paraná. For this purpose, eight samples of lettuce (Lactuca sativa) and eight samples of chicory (Cichorium intybus) were collected and analyzed. The analysis was performed at the Laboratory for Extension and Research in Enteroparasitosis L.E.P.En. Cysts of Endolimax nana, Entamoeba coli, Entamoeba histolytica, Balantidium coli and Strongyloides stercoralis larvae, Hookworm larvae and eggs, and eggs of Ascaris spp were found in the samples. These results demonstrate the need for hygienic-sanitary measures, aimed at educating the community, enabling the improvement in living conditions of the population.
\end{abstract}

Keywords: Intestinal parasites. Vegetables. Search. Public health.

${ }^{1}$ Farmacêutica, Pós-graduanda: Residente em Análises Clínicas pelo Departamento de Patologia, Análises Clínicas e Toxicológicas da Universidade Estadual de Londrina (UEL), Londrina, PR, Brasil.

${ }^{2}$ Farmacêutica, Pós-graduanda: Residente em Análises Clínicas pelo Departamento de Patologia, Análises Clínicas e Toxicológicas da Universidade Estadual de Londrina (UEL), Londrina, PR, Brasil.

${ }^{3}$ Farmacêutico, Pós-graduando: Residente em Análises Clínicas pelo Departamento de Patologia, Análises Clínicas e Toxicológicas da Universidade Estadual de Londrina (UEL), Londrina, PR, Brasil.

${ }^{4}$ Graduanda em Farmácia pela Universidade Estadual de Londrina, PR, Brasil.

${ }^{5}$ Farmacêutica, Mestre em Patologia Experimental, Docente do Departamento de Patologia, Análises Clínicas e Toxicológicas da Universidade Estadual de Londrina (UEL), Londrina, PR, Brasil.

${ }^{6}$ Biomédico, Doutor em Patologia Experimental, Docente do Departamento de Patologia, Análises Clínicas e Toxicológicas da Universidade Estadual de Londrina (UEL), Londrina, PR, Brasil.

${ }^{7}$ Biomédico, Especialista em Protozoologia e Helmintologia, Docente do Departamento de Patologia, Análises Clínicas e Toxicológicas da Universidade Estadual de Londrina (UEL), Londrina, PR, Brasil. 


\section{Introdução}

As parasitoses intestinais estão entre as doenças mais comuns mundialmente, representando assim um grave e constante problema para a saúde pública, principalmente em áreas rurais e população de baixa renda. São de difícil solução e a sua prevalência é muito alta em locais onde condições de vida e de saneamento básico são insatisfatórias ou até mesmo inexistentes. Tais infestações podem ser facilitadas e a sua reincidência predisposta tanto pela falta de conhecimento de condições básicas de higiene quanto pelo descuidado na preparação de alimentos (ANDRADE et al., 2010).

Alimentos de origem vegetal irrigados com água contaminada ou efluente não tratado são grandes veiculadores de microrganismos, os quais são capazes de gerarem tais parasitoses em graus diferenciados, podendo até levar à morte. Assim, o consumo desses alimentos in natura, que vem aumentando com a busca cada vez maior por hábitos alimentares saudáveis, aumenta a possibilidade de transmissão das parasitoses, já que a contaminação pelos parasitas mais comuns é, em grande parte, pelo ciclo fecal/oral. Tal contaminação pode ter origem em várias etapas da manipulação dessas hortaliças, como na horta, através da utilização de adubos orgânicos contaminados com dejetos fecais ou da irrigação com água de má qualidade; no transporte em caminhões abertos; no acondicionamento em caixa sujas; no armazenamento em locais inadequados ou até mesmo na manipulação nos pontos de venda. (NEVES; FILIPPIS, 2010).

A análise parasitológica das hortaliças é de grande importância para a saúde pública, já que fornece dados sobre o estado de cultivo dessas hortaliças e permite o controle sobre o cultivo, armazenamento, transporte e preparação desses alimentos. Assim, essa análise tem como principal objetivo identificar os parasitas intestinais do homem, através da visualização de diferentes formas parasitárias eliminadas nas fezes (COELHO et al., 2001; GELLI et al., 1979).

Levando em consideração a importância dessas análises, esse trabalho teve como objetivo avaliar a ocorrência de parasitas intestinais em alfaces e almeirões, comercializadas em feira livre e supermercado de Londrina, Paraná, já que são hortaliças de consumo comum na sociedade, tornandose assim, um fácil meio de veiculação de parasitoses.

\section{Materiais e Métodos}

\section{Descrição das amostras}

A pesquisa foi realizada com maços de alface e maços de almeirão, comprados em uma feira livre e um supermercado, localizados em diferentes regiões da cidade de Londrina, Paraná, durante dois meses. As verduras foram escolhidas de forma aleatória, estabelecendo como unidade, tanto para a alface quanto para o almeirão, o pé em maço, independente do peso e do tamanho, porém, de boa qualidade. Foram analisados 1 pé de alface e 1 pé de almeirão por semana, totalizando 16 amostras, acondicionadas em sacos plásticos assépticos e descartáveis e encaminhadas ao Laboratório de Extensão e Pesquisas em Enteroparasitoses - L.E.P.En., localizado no Ambulatório de Hospital de Clínicas (AHC), da Universidade Estadual de Londrina (UEL) para análise.

\section{Análise parasitológica}

O processamento das amostras e a análise parasitológica foram realizados como previamente descrito por Guimarães et al. (2003), com algumas modificações.

As verduras foram lavadas em duas etapas:

a) primeira etapa: dentro do saco plástico adicionou-se $250 \mathrm{~mL}$ de água destilada e agitada manualmente durante 1 minuto;

b) segunda etapa: as verduras foram desfolhadas, submersas e pinceladas individualmente dentro de uma cuba plástica contendo $250 \mathrm{~mL}$ de água destilada, juntamente com $1 \mathrm{~mL}$ de detergente neutro. As soluções das duas lavagens foram 
filtradas em gaze cirúrgica em 8 dobras, sobre um cálice de sedimentação de plástico e submetidas aos métodos qualitativos de sedimentação espontânea descrita por Hoffman, Pons e Janer (1934) e a de centrífugo-flutuação em sulfato de zinco descrita por Faust et al. (1939). As amostras foram coradas em solução de Lugol e examinadas em duplicata com auxílio do microscópio óptico nos aumentos de $10 x$ e $40 x$.

\section{Resultados e Discussão}

Foram analisadas oito amostras de cada espécie de verdura e não foi observada diferença na contaminação por parasitas intestinais entre as hortaliças comercializadas na feira livre e no supermercado, levando-se a acreditar na possibilidade de que essas hortaliças tenham a mesma origem. Em estudos semelhantes realizado em Gurupi, estado do Tocantins, por Silva e Gontijo (2012), e em Cuiabá, no Mato Grosso, por Alves, Cunha Neto e Rossignoli (2013), foi também evidenciado um elevado número de contaminação em alfaces. Em Gurupi, do total de 110 amostras, $60 \%$ estavam contaminadas (SILVA; GONTIJO, 2012); em Cuiabá, de 45 amostras analisadas, 66,7\% mostraram-se contaminadas (ALVES; CUNHA NETO; ROSSIGNOLI, 2013).

$\mathrm{Na}$ análise parasitológica feita nas alfaces e almeirões, houve predominância de cistos de Endolimax nana em 100\% das amostras, seguidos de 87,5\% de larvas de Ancilostomídeos, 87,5\% de cistos de Balantidium coli, Entamoeba histolytica, $75 \%$ de larvas de Strongyloides stercoralis e 62,5\% de Entamoeba coli nas alfaces e 50\% nos almeirões. Com menor ocorrência, foram identificados os ovos de Ascaris spp. em 37,5\% nos almeirões e 25\% nas alfaces (Tabela 1).

Tabela 1 - Frequência de enteroparasitas encontradas em amostras de alface e almeirão.

\begin{tabular}{|c|c|c|c|c|}
\hline Hortaliça & \multirow[t]{2}{*}{ Alface } & \multirow[t]{2}{*}{$\%$} & \multirow[t]{2}{*}{ Almeirão } & \multirow[t]{2}{*}{$\%$} \\
\hline Parasita & & & & \\
\hline Endolimax nana & 8 & $100 \%$ & 8 & $100 \%$ \\
\hline Entamoeba histolytica & 7 & $87,5 \%$ & 7 & $87,5 \%$ \\
\hline Entamoeba coli & 5 & $62,5 \%$ & 4 & $50 \%$ \\
\hline Ancilostomídeos & 7 & $87,5 \%$ & 7 & $87,5 \%$ \\
\hline Strongyloides stercoralis & 6 & $75 \%$ & 6 & $75 \%$ \\
\hline Balantidium coli & 7 & $87,5 \%$ & 7 & $87,5 \%$ \\
\hline Ascaris lumbricoides & 2 & $25 \%$ & 3 & $37,5 \%$ \\
\hline
\end{tabular}

Fonte: Autores.

Apesar do E. nana e E. coli serem considerados protozoários comensais, sua ocorrência é indicativo de transmissão fecal-oral, o que pode determinar a transmissão de agentes parasitários patogênicos. Sendo assim, o diagnóstico e a descrição destes comensais são importantes, a fim de se programar medidas profiláticas para evitar infecção devido à contaminação oro-fecal de amebas patogênicas, como a E. histolytica, e outros gêneros de parasitas (BELOTTO et al., 2011). Encontrou-se neste estudo, também, a a infecção por Ancilostomídeos é assintomática e o prevalência de Ancilostomídeos em 100\% das amostras. A ancilostomíase ocorre em $20 \%$ a $25 \%$ da população mundial. No Brasil a prevalência é variável, sendo mais comum na zona rural, onde a habitação e o peridomicílio são os locais que oferecem maior possibilidade de contaminação, já que é necessário contato com as larvas presentes no solo. Acometendo mais adolescentes e adultos (diferentemente das protozooses que acometem mais frequentemente crianças), na maioria das vezes 
quadro clínico dependerá do número e da espécie do verme e das condições do hospedeiro. As infestações agudas e maciças por ancilóstomos podem resultar em fadiga, fraqueza, dores abdominais e diarreia sanguinolenta. As infestações crônicas resultam em anemia, palidez, apatia e tontura, devido à ação espoliadora do parasita no intestino humano (BLOOMFIELD, 2001; MELO et al., 2004; NEVES et al., 2011).

O Balantidium coli é normalmente um protozoário comensal da luz intestinal de suínos. Pode parasitar os humanos quando ocorre uma lesão na mucosa do colo e do ceco, ocorrendo uma infecção secundária, já que não é capaz de penetrar sozinho nas mucosas intactas (NEVES et al., 2005).

Os resultados encontrados neste estudo talvez resultem de atos que propiciam a contaminação nas diferentes etapas produtivas, incluindo o plantio, o tipo de água para irrigação, adubos utilizados, colheita, transporte, além da manipulação das hortaliças pelos produtores ou nos estabelecimentos comerciais. Esses dados corroboram com a literatura que tem demonstrado a presença de parasitas intestinais como um grave problema de saúde pública no Brasil, como encontrado por Neres et al. (2011), no município de Anapólis, Goiás, Gregório et al. (2012), na Região Leste de São Paulo e Vieira et al. (2013), no sul do Rio Grande do Sul.

Um estudo realizado por Rocha et al. (2003), avaliou o material utilizado para adubar as hortaliças e observou um elevado índice de contaminação nas hortaliças, o que pode significar uma importante fonte de contaminação das mesmas, já que o tempo de sobrevivência dos ovos no meio favorece sua viabilidade até o momento do consumo destas verduras.

Apesar de as condições urbanas de saneamento e moradia serem satisfatórias em regiões bem urbanizadas, como a região Sul do Brasil, o acentuado êxodo rural e o acelerado crescimento de favelas e ocupações de áreas irregulares aumentam as chances de exposição às parasitoses.

\section{Conclusão}

Com base nos dados obtidos neste estudo, conclui-se que as amostras de alfaces e almeirões comercializadas na cidade do norte do Paraná, apresentam formas parasitárias humanas e/ou animais, sendo assim, um importante veículo de transmissão de parasitas intestinais. Há uma necessidade de medidas que propiciem uma melhora na qualidade higiênico-sanitária, e previamente ao consumo, associar uma desinfecção das hortaliças consumidas in natura, para minimizar os riscos de transmissão de enteroparasitas, pois a lavagem simples não reduz a contaminação.

\section{Referências}

ALVES, A. S.; CUNHA NETO, A.; ROSSIGNOLI, P. A. Parasitos em alface-crespa (Lactuca sativa L.), de plantio convencional, comercializada em supermercados de Cuiabá, Mato Grosso, Brasil. Revista de Patologia Tropical, Goiânia, v. 42, n. 2, p. 217-229, 2013.

ANDRADE, E. C.; LEITE, I. C. G.; RODRIGUES, V. O.; CESCA, M. G. Parasitoses intestinais: uma revisão sobre seus aspectos sociais, epidemiológicos, clínicos e terapêuticos. Revista APS, Juiz de Fora, v. 13, n. 2, p. 231-240, 2010.

BELLOTO, M. V. T., SANTOS JUNIOR, J. E.; MACEDO, E. A.; PONCE, A.; GALISTEU, K. J.; CASTRO, E.; TAUYR, L. V.; ROSSIT, A. R. B.; MACHADO, R. L. D. Enteroparasitoses numa população de escolares da rede pública de ensino do Município de Mirassol, São Paulo, Brasil. Revista Pan-Amazônica de Saúde, Ananindeua, v. 2, n. 1, p. 37 - 44, 2011.

BLOOMFIELD, S. F. Preventing Infectious diseases in the domestic setting: a risk-based approach. American Journal of Infection Control, Saint Louis, v. 29, p. 207-212, 2001. 
COELHO, L. M. P. S.; OLIVEIRA, S. M.; MILMAN, M. H. S. A.; KARASAWA, K. A.; SANTOS, R. P. Detecção de formas transmissíveis de enteroparasitas na água e nas hortaliças consumidas em comunidades escolares de Sorocaba, São Paulo, Brasil. Revista da Sociedade Brasileira de Medicina Tropical, Uberaba, v. 34, n. 5, p. 579-482, 2001.

FAUST, E. C.; SAWITZ, W.; TOBIE, J.; ODOM, V.; PERES, C.; LINCICOME, D. R. Comparative efficiency of various techniques for the diagnoses of protozoa and helminthes in feces. The Journal of Parasitology, Saint Louis, v. 25, n. 3, p. 241262, 1939.

GELLI, D. S.; TACHIBANA, T.; OLIVEIRA, I. R.; ZAMBONI, C. K.; PACHECO, J. A.; SPITERI, N. Condições higiênico-sanitárias de hortaliças comercializadas na Cidade de São Paulo, SP, Brasil. Revista do Instituto Adolfo Lutz, São Paulo, v. 39, p. 37-43, 1979.

GREGÓRIO, D. S.; MORAES, G. F. A.; NASSIF, J. M.; ALVES, M. R. M.; CARMO, N. E.; JARROUGE, M. G.; BOUÇAS, R. I.; SANTOS, A. C. C.; BOUÇAS, T. R. J. Estudo da contaminação por parasitas em hortaliças da Região Leste de São Paulo. Science in Health, São Paulo, v. 3, n. 2, p. 96-103, 2012.

GUIMARÃES, A. M.; ALVES, E. G. L.; FIGUEIREDO, H. C. P.; COSTA, G. M.; RODRIGUES, L. S. Frequência de enteroparasitas em amostras de alface (Lactuca sativa) comercializadas em Lavras, Minas Gerais. Revista da Sociedade Brasileira de Medicina Tropical, Uberaba, v. 36, p. 621-623, 2003.

HOFFMAN, W. A.; PONS, J. A.; JANER, S. L. The sedimentation-concentration methods in Schistosomiasis mansoni. The Puerto Rico Journal of Public Health and Tropical Medicine, Puerto Rico, v. 9, p. 281-298, 1934.

MELO, M. C. B.; KLEN, V. G. Q.; MOTA, J. A. C.; PENNA, F. J. Parasitoses intestinais. Revista Médica de Minas Gerais, Belo Horizonte, v. 12, n. 1, supl. 1, p. 3-12, 2004.
NERES, A. C.; NASCIMENTO, A. H.; LEMOS, K. R. M.; RIBEIRO, E. L.; LEITÃO, V. O.; PACHECO, J. B. P.; DINIZ, D. O.; AGMF AVERSI-FERREIRA, R.; AVERSI-FERREIRA, T.; A. Enteroparasitos em amostras de alface (Lactuva sativa var. crispa), no município de Anápolis, Goiás, Brasil. Bioscience Journal, Uberlândia, v. 27, n. 2, p. 336-341, 2011.

NEVES, D. P.; FILIPPIS, T. Parasitologia básica. 2. ed. São Paulo: Atheneu, 2010.

NEVES, D. P., MELO, A. L.; LINARDI, P. M.; VITOR, R. W. A. Parasitologia humana. 11. ed. São Paulo: Atheneu, 2005.

NEVES, D. P., MELO, A. L.; LINARDI, P. M.; VITOR, R. W. A. Parasitologia humana. 12. ed. São Paulo: Atheneu, 2011.

ROCHA, R. E. M.; PIMENTEL, M. S.; ZAGO, V. C. P.; RUMJANEK, N. G.; DE-POLLI, H. Avaliação de biossólido de águas servidas domiciliares como adubo em couve. Pesquisa Agropecuária Brasileira, Brasília, v. 38, n. 12, p. 1435-1441, 2003.

SilVA, M. G.; GONTIJO, E. E. L. Avaliação parasitológica de alfaces (Lactuca sativa) comercializadas em supermercados e feiras livres do município de Gurupi, Tocantins. Revista Cientifica do ITPAC, Araguaína, v. 5, n. 4, out. 2012.

VIEIRA, J. N.; PEREIRA, C. P.; BASTOS, C. G. G.; NAGEL, A. S.; ANTUNES, L. VILLELA, M. M. Parasitos em hortaliças comercializadas no sul do Rio Grande do Sul, Brasil. Revista de Ciências Médicas e Biológicas, Salvador, v. 12, n. 1, p. 4549, 2013. 
